\title{
Doubts in business communication - can we transform perception into message
}

\section{Jovanovic Dragana ${ }^{14}$, Baltezarevic Vesna ${ }^{15}$, Baltezarevic Radoslav ${ }^{16}$}

\begin{abstract}
The communication potential of virtual technology is widely recognized. Here, we review current problems related to developing, evaluating, or using virtual technology in business communication. Business uses of digital technology are broadly understood, but some of the questions we discussed here raise confusion about virtuality and reality, mental illusions, intransitive condition, and impassable boundaries of virtuality - embodied, sensual, singular subjectivity. Virtual communication is far beyond using computer technology, as we know it today. However, we all should be aware of confusion related to computer-mediated perception. From individual perception, communication sometime moves toward contemplative abstraction, which tends to forget its precondition: the original, mediated perceptual-sensuous immediacy of physical existence in the world. Perception is the unmediated directness, original, non-objected participation of organism in the universe. That is existence in unity with things, coexistence with the world. Original or phenomenological, sensory perception is not seen as a sign that the object emits and which informs us about it, or as structures of our brain centers or translation energy pulses in the respective forms - all these ideas are subsequent, second level or secondary metal structures. Perception is not noticed, but modus vivendi, manner of existence, the direct participation in the universe. This is the metaphysical meaning of perception, which doesn't deny the necessity of action, forces us to treat perceptions as a message, but it is secondary and derived meaning that we cannot legitimately absolutize.
\end{abstract}

KEY WORDS: virtual technology, communication, reality, body, perception, message, digital, media

JEL: $A 12$

UDC: 005.57

659.23:[007:004

COBISS.SR-ID 219518220

\footnotetext{
${ }^{14}$ Corresponding author,Faculty of Culture and Media, Megatrend University, Belgrade, email: djovanovic@ nezbit.edu.rs

${ }^{15}$ Faculty of Culture and Media, Megatrend University, Belgrade, e-mail: vesnabal@gmail.com

${ }^{16}$ Alma Mater Europaea Belgrade, e-mail: trilliongarden@yahoo.com
} 


\section{Introduction}

Modern man is experiencing a radical change of attitude towards time due to domination of information technologies as parameters that control_our lives. Today, business is characterized by speed feverishness, hyperactivity, impatience, impulsiveness, dispersal, and fragmentation of attention due to the overwhelming abundance of various unrelated and unstructured data, information, and content. Quantitative criteria dominate the qualitative productivity of communication, and the depth and integrity are lost to scattering and extensions. Speed, quantity and dispersal inevitably result in superficiality, imperfection, and violence against sensitive, delicate rhythms of cognitive, emotional, social and creative individual ripening. To be manifested and accomplished, communication potentials and dispositions require a continuing and long-term learning and practicing, uncompromising (self) discipline, focus, attention, and dedication, and ignoring many technological and media distractions, of which the most powerful is, of course, the Internet. As an interactive medium, the Internet can encourage communication, but if it can capture time and monopolize the attention and energy, the message will stay stuck at the level of spontaneous expressivity, as a prerequisite, but not the essence of communication.

Due to its multi dimensionality and interactivity, the Internet is mass media with the greatest potential for socializing. Contrary to the claims of researchers that the media is a secondary agent of business socialization, several surveys put it among the primary agents. This is the first time in history that a medium appeared in the position of the flywheel of socialization. At this point, we face a basic problem of Internet usage in business communication: are managers skilled enough to make a difference between reality and virtuality, and between information and message?

Modern information and technology enable creating virtual worlds so alike to the real world, but development in neuroscience and new techniques of brain research show us how our senses and emotions generate, and give new force to idealist and constructivist theories of sensory perception. By the logic of such experiments, we easily reach their idealistic and solipsistic consequence - the world is our notion. All we see and feel in our lives buildings, people, cities, places - all what we can taste and hear is shaped in our brains, but does not exist there. In fact, what exist are electrical impulses that form our perceptions. Our cognition of the world is just an interpretation of certain stimulus create in our brains. So, what about virtual marketplace and virtual relations among businessman, what about immaterial contacts and understanding of computer mediated message?

Interaction between man and computer is so improved, sophisticated, and convincing that we begin to believe that machines can think and talk to us, and that simulation is actually real and independent of consciousness. We begin to believe that what produces the thought is just as real as what exists beyond her, that the simulation of virtual larva of a butterfly coming out is the real phenomena. There must be some kind of intoxication of power in the belief that one can master creation, that with the technological means we can be equated with God in the power of producing the overall appearance. 


\section{Communication or mental illusion}

Communication requires, above all, attention and learning. The highest form of learning is a heuristic - insightful learning that requires creativity and the other forms of learning, such as repetition (practice), instrumental learning, and learning by model. Communication knowledge cannot be create as a single field that has not previously learned the basics, and trained in the techniques and skills. Important factors for successful communication are methods used in the course of learning. Mastering communication knowledge means that we first need to learn, and learn means that we need to pay attention to the information. Every voluntary act requires attention, but attention capacity is limited. We can only process a certain amount of information at the same time. Exactly how much is not known, and depends on the individual, but in fact, a good portion of our limited reserves of attention is devoted to the tasks necessary for daily life. In life, learning in any symbolic sphere is only a fraction of available amount of attention. Therefore, the time dedicated to developing communication skills is valuable.

However, behind computers are individuals - organisms of flesh, blood, and bodies with consciousness who are networked, i.e., intersubjectivity. A people before technology, before creating and entering the virtual world, before networking, communicate biologically by their bodies, where, or proceeding from, they live mentally by their spirits and affectively by their hearts. They themselves are not machines, not robots and avatars, not products of anyone's imagination, digital arts and construction. As much as we try, the virtual world will always be deficient in relation to reality, primarily because of the intransitive condition and impassable boundaries of virtuality - embodied, sensual, singular subjectivity.

Here is the biggest problem of virtual communication: virtual worlds are unable to mobilize the entire subjectivity, neither on perceptual, cognitive, or emotional levels. First of all, they do not mobilize all five senses; therefore, neither of their complex interactions constitutes the integrity of perceptual experience. In addition to achieving perceptual integrity, individual senses exercise mutual influence, intensifying, deepening and sharpening one another. For example, a touch enhances vision; hearing or vision enhances the taste; smell enhances vision (both external and internal in the form of mental images), etc. However, in front of computer monitor, the human body is passive, inert, and solid. In computer usage only fingers are active, often only two fingers! The lack of sensory diverts stimuli and responses, blocking associative paths and processes, e.g., sense of smell the fastest evokes the most vivid memories, the movement causes a change in spatial and cognitive perspective, introducing different moods, and feelings; thus, it facilitates creative breakthroughs. As stated by many businessma: moving, traveling, or changing interior or landscape (i.e. diverse and dynamic sensory stimulation) significantly favored creativity and communication potentials. 


\section{Theoretical framework}

Virtual worlds promoted on-screen, even if they were in 3D or in the near future, with the addition of olfactory and tactile ingredients, all in an effort to achieve a more complete realism, eliminate predictability, spontaneous intentionality of individual perceptions, feelings, and consciousness. Their embeddedness is an_intimate, unique, and integrated sense of self. Again, that is in response to the question of what is the reality that, although masked and forgotten, lies at the heart of any virtuality. What is the fundamental reality that ontologically precedes consciousness, thought, imagination, knowledge, science, and technological innovation? It is the body, namely the individual, private body with his nervous and sensory systems. The foundation of all mental functions is sensory perception and corporeality. From individual perception, communication moves toward contemplative abstraction, which tends to forget its precondition: the original, mediated perceptualsensuous immediacy of physical existence in the world.

Original or phenomenological, sensory perception is not seen as a sign that the object emits and which informs us about it, or as structures of our brain centers, or translation energy pulses in the respective forms. All these ideas are subsequent, second level, or secondary mental structures. Perception is not notice, but modus vivendi, manner of existence, the direct participation in the Universe. It is "the base for interpretation and communication in general and therefore cannot be the interpretation or communication itself" (Marcel, 1934). This is the metaphysical meaning of perception, which does not deny the necessity of action, forcing us to treat perceptions as a message, but it is secondary and derived meaning that we cannot legitimately absolutize.

First, directness of thought and every mental ascent is perception. The movement of thought is not possible without interaction, or rather a collision with an element that interrupts her temporary state of rest. In a word, dialectic does not exist without the existential shock. It is wrong, however, to think that the sensory perception is necessary, but only a first step and initiator of complex cognitive actions that go beyond it and leave it behind. The whole thought and elaboration imbued with physical and sensory dimensions, which is reflected in the metaphorical nature of language, as well as the symbolic nature of all human cultural production, especially the arts. All we experience is the result of a set of perceptions that involves all our senses and recognizes the importance of things, and which arises from the interpretation that we give to any moment of this protean agglomeration. The entire set of symbolic activity comes from sensory experiences, reflecting, reflects, and transforms. There is continuity between perception and intelligence. But, what about communication without the physical body? Bodily phenomena, such as muscle activity, breathing, posture, muscle tension, nonverbal communication, etc., affect the mental representation and rivers. Intervention on a somatic level affects the psychological level, and vice versa. These relationships simultaneously include: (a) mental representations, (b) physiological excitement, (c) communicative impact, and (d) feed-back mechanism from which we learn. Whole perception includes the environment and does not recognize the body as bounded within its borders, but as a system open to other systems. That presence, which is open to participation, is found in the word exist.

In the virtual world, we are not responsible for the lives of others. Our_miscalculation, clumsiness, and negligence will not run hurt anyone. In the virtual world, there are no actions and consequences, only the procedures, actions, and effects. In the virtual world, nothing collides realistically. There is no finality, end, pain, anxiety, guilt, remorse, shame, 
and borderline situations. There is, therefore, no work on the matter, on the other, on itself; there are no inner psychic conflicts, catharsis, transformation, and sublimation. There is density, opacity, but no resistance to the reality that encourages exploration, combat, and self-transcendence. Acquisition and improvement of skills in solving the tasks set out in virtual scenarios, hire at least our body, thus, in fact, even less, our character, ethics, emotions (also anger and frustration when we fail at any given virtual task). Human body is the place where desire is born, where we encounter experiences, ease, grace, joy, harmony, sexuality, or weight, nausea, resistance limits and inertia. None of this has anything to do with abstract, visual virtuality based on the performance of the body build in the digital world. Illegitimate identification of scientific and technological analysis, deconstruction and reconstruction of original reception, de-realized fundamental experience of reality and opens the door for her absolute virtualization.

Perception and cognition are, with the second instance, the secondary standpoints that take place in a closed circuit of their own state of mind, or more accurately, state of the brain. The same goes for love, aesthetic enjoyment, evaluation and so on. But perception, thought, love, and evaluation are intentional acts that affirm the transcendence of beings (objects of intentionality) in relation to themselves. World, being, surprising, inspiring, fulfills, baffles, limits, and pressures exists only in transcend states of consciousness (the brain). With extreme immanentism, by which the world is a product of neurological organization, the technology can support through virtual simulation, a powerful illusion of unreality - virtuality of reality itself. The natural man, the concrete individual, or whole organism with the senses and consciousness, which breathes, eats, excretes, tired and resting, which is related to biorhythm, weather conditions, physiological cycles, fare behind the virtual worlds that are sometimes taught in oblivion as its reality. The same sensible, perceptual and emotional human body experiences its original, irreducible sources of reality as unreal and illusory. We live in a world that our senses can only partially explore. Some senses naturally became insignificant in technicalized world. Our senses are idle. We do not come to important discoveries by them, but by machines. However, embodiment or incarnation (incarnated subjectivity) is a complex experience. Observing specifically physical experience, we discover that it is far from reality made up of different parts of the external set in relation to one another.

\section{Conclusion}

One of threatening factors that dominate our lives and perception of reality is speed. Time is structural given existence, and the speed is a structural dimension of technology. As the technology has won our lives, speed has become a business modus vivendi. Slow pace and irritating experience intense social condemnation: treated as a nonchalant, relaxed, lazy, idle, unproductive, inefficient, and so on. In a society obsessed with visible and measurable results, introspective leisure and regeneration powers are necessary for the reconstruction of conceptual and experiential inspiration for understanding the problem from a different perspective, understand as laziness, unauthorized slowness and immobility, lost time - nothing. Communication is not considered work, because work is measured by industrial, technological and economical parameters. Creative contemplation, mindfulness stands against neurotic activism as a loss of self to the outside world - dominated feverish techno time. Radical change in attitude towards time occurs with industrialization and climaxes with the information-technology revolution, which, in conjunction with the logic 
of financial markets, introduced so-called dictatorship of "real time". Speed is a decisive, instant reaction to signals transmitted on the market. Internet brought the process of acceleration to a boil. Preferred speed, immediacy of execution for machines and computers become a growing urgency for a man. "Life has become hysterical series of crowded moments" (Eriksen, 2003).

The term real time is actually a misnomer, and is based on a narrow conception of time and reality. It is unrealistic, chopped, successive time contrary to the experience of life, learning are creative property, said Bergson. Duration of existential, qualitative, unique, spontaneous, continuous and unpredictable flow is misunderstood. Objectified time creates the illusion of triumph over time (and the area). Regard this, we experienced the urge for existential ubiquity: to be ubiquitous, ongoing, have as many friends and information as we can, links and contents also, of which a tenth part has completed. This leads to the opposite of the desired outcome, i.e., triumph of man over time and various aspects of the pathology of temporality. Continuity, integrity and purposeful learning efforts are just a testimony to man's ontological needs that combat fragmentation, dispersion and instantism. Structural features of technology and financial economy spilled over into all spheres of society, imposing the same imperative of instantaneity. Rush became the dominant mode of planning and organizing collective and individual life. There is excess and uncontrolled increasing amount of information available.

In business world more information fights for a shorter time, which leads to compression of information in shorter time frames. Compression of time means shorter time units by which we measure how much time we need to do or accomplish something. But, the human brain has not sped up its functions. Information explosion caused cognitive to implode in disintegration, disorder, and return to the simpler, cruder forms of thought and experience. The prevailing cognitive and emotional automatisms according to the scheme S-R (stimulus - response) lead to routinized opinion, which is opposite of organized, complex, challenging and integrative processes of creative learning. Speed clicking and surfing is a drastic step with its rhythm and dynamics: acceptance, processing, integration and understanding of data; thinking and reasoning; shaping and creative experience, and finally - communication process.

Speed impacts higher cognitive connection functions of linkage, analysis, synthesis, complex thought, gaining a broader perspective, interpretation and metacognitive dimension filtering, priority setting, monitoring, evaluation, and correction of personal opinion. Technological and informational speed, which has become a cultural and mental matrix, influences communication, so we point out some of the key conditions, factors and dimensions of creativity, requires a different temporality, that is, continuity, development, slow, patience. First of all, to be manifested and accomplished, communication potential and dispositions required: long-term, continuous and regular communicating; unconditional (self) discipline; focus of attention and dedication, and ignoring or many (technological and media) distracters. Visible part of the communication process is only the tip of the iceberg, which is preceded by a huge invisible ineffective, unpredictable, uncertain process. Dimension of life, where communication takes place, corresponds to a different state of consciousness that transcends succession of moments and resist decline in fragments as a unit of time alienated of existence. 


\section{References}

[1] Ackerman, D.(1991). A Natural History of the Senses. New York: Knopf Doubleday Publishing Group

[2] Gibson, W.(1986). Neuromancer. New York: Ace.

[3] Marcel, G.(1934) .Etreetavoir. Paris: Aubier

\section{Article history:}

- Received 18 September 2015

- Accepted 20November 2015 